\title{
Serum $7 \alpha$-hydroxy-4-cholesten-3-one concentrations in the evaluation of bile acid malabsorption in patients with diarrhoea: correlation to SeHCAT test
}

\author{
S Eusufzai, M Axelson, B Angelin, K Einarsson
}

\begin{abstract}
The synthesis of bile acids is regulated by a homeostatic mechanism in which bile acids returning to the liver from the intestine inhibit their own synthesis. Serum concentrations of the bile acid intermediate $7 \alpha$-hydroxy-4cholesten-3-one reflect the rate of bile acid synthesis whereas bile acid malabsorption can be determined by the SeHCAT test. This study was done to evaluate the correlation between the two tests in humans. Twenty eight patients with chronic diarrhoea were included in the study. Fasting serum was collected for the determination of $7 \alpha$-hydroxy4-cholesten-3-one, and on the same day the $\gamma$ emitting bile acid analogue SeHCAT was given orally and its fractional catabolic rate assessed by repeated external counting over the upper abdomen during the next seven days. There was a highly significant positive correlation between the two tests $\left(R_{s}=0.80, p<0.001\right)$. The results show a close relation between intestinal loss and hepatic synthesis of bile acids and imply that analysis of $7 \alpha$-hydroxy-4cholesten-3-one in serum should now be evaluated as a possible convenient method for assessing bile acid malabsorption in patients with diarrhoea.
\end{abstract}

(Gut 1993; 34: 698-701)

Bile acids are necessary for the absorption of dietary fats and cholesterol from the intestine. They are produced in the liver from cholesterol, stored in the gall bladder, and are excreted into the intestine during meals. ${ }^{1}$ More than $95 \%$ of the bile acids are then reabsorbed, the major portion by active uptake in the distal ileum. Malabsorption of bile acids can induce diarrhoea, a symptom common to many intestinal diseases. In recent years, the SeHCAT test has been used for the determination of bile acid malabsorption. Selenium-75 homocholic acid taurine $\left({ }^{75} \mathrm{SeHCAT}\right)$ is a synthetic analogue of the natural conjugated bile acid taurocholic acid with the $\gamma$ emitting ${ }^{75} \mathrm{Se}$ in the side chain. ${ }^{23}$
SeHCAT, which is resistant to deconjugation by intestinal bacteria, otherwise behaves like taurocholic acid with regard to enterohepatic cyling, hepatic handling, active absorption from the terminal ileum, faecal excretion, and overall turnover in the enterohepatic circulation..$^{2-6}$

The rate of synthesis of bile acids is determined by a homeostatic mechanism regulated by the amount of bile acids returning to the liver from the intestine (negative feed back). Thus patients with significant bile acid malabsorption can be expected to have an increased production of bile acids. Cholesterol $7 \alpha$-hydroxylase is considered to catalyse the rate limiting reaction in the biosynthesis of bile acids, converting cholesterol into $7 \alpha$-hydroxycholesterol. ${ }^{7}$ This sterol is then converted into bile acids via the intermediate $7 \alpha$-hydroxy-4-cholesten-3-one (Fig 1). Recent studies have shown that plasma concentrations of $7 \alpha$-hydroxy-4-cholesten-3-one reflect the cholesterol $7 \alpha$-hydroxylase activity and bile acid production in the human liver. ${ }^{89}$

The aim of this study was to determine whether there is any correlation between the serum concentration of $7 \alpha$-hydroxy-4-cholesten3-one and the result of the SeHCAT test. If so, analysis of serum $7 \alpha$-hydroxy-4-cholesten-3-one may eventually be used to identify bile acid malabsorption in humans.

\section{Methods}

\section{PATIENTS AND CONTROLS}

The study comprised 28 patients with diarrhoea who were consecutively referred for the SeHCAT test (Table). All of the patients had undergone extensive investigations to evaluate their intestinal function. After a basal clinical and laboratory evaluation excluding heart, kidney, and liver disease, they were followed closely as outpatients and allowed to continue their normal diet. Any antidiarrhoeal drugs were stopped at least three days before the SeHCAT test. On the morning of the SeHCAT test the patients had been fasting overnight. Blood
Department of Medicin

Hospital, Sweden

S Eusufzai

K Einarsson

Department of Clinical Chemistry, Karolinska Hospital, Karolinska Institute, Stockholm, Sweden

M Axelson

Correspondence to: Dr Sharif Eusufzai, Department of Medicine, Huddinge University Hospital, S-141 86 Huddinge, Sweden.

Accepted for publication 15 September 1992

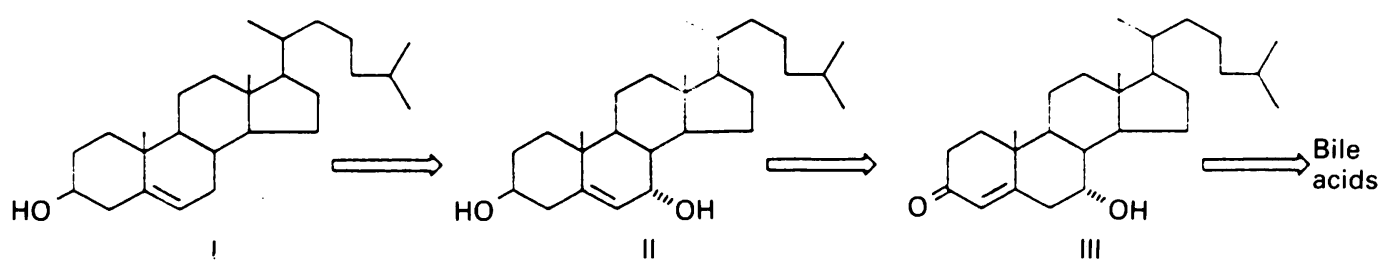

Figure 1: A simplified scheme for the conversion of cholesterol (I), into bile acids through $7 \alpha-$ hydroxycholesterol (II), and $7 \alpha$-hydroxy-4-cholesten-3-one (III). The first reaction is catalysed by cholesterol $7 \alpha-$ hydroxylase. 
Data on patients with chronic diarrhoea studied with selenohomocholic acid taurine (SeHCAT) test and serum measurements of $7 \alpha$-hydroxy-4-cholesten-3-one

\begin{tabular}{|c|c|c|c|c|c|}
\hline Patient & $\begin{array}{l}\text { Age/sex } \\
(y)\end{array}$ & $\begin{array}{l}\text { Duration } \\
\text { of } \\
\text { symptoms } \\
(y)\end{array}$ & $\begin{array}{l}\text { SeHCAT } \\
\text { FCR } \\
\text { (pools/ } \\
\text { day) }\end{array}$ & $\begin{array}{l}\text { 7a-hydroxy- } \\
\text { 4-cholesten-3- } \\
\text { one }(\text { ng/ml })^{8}\end{array}$ & Additional comments \\
\hline 1 & $32 / \mathrm{F}$ & - & 0.035 & 7 & \\
\hline 2 & $48 / \mathrm{F}$ & 8 & 0.055 & 11 & Cholecystectomy \\
\hline 3 & $46 / F$ & 2 & 0.062 & 8 & \\
\hline 4 & $68 / \mathrm{F}$ & 10 & $0 \cdot 130$ & 45 & Radiation therapy \\
\hline 5 & $26 / M$ & 1 & $0 \cdot 153$ & 7 & \\
\hline 6 & $44 / F$ & 4 & $0 \cdot 159$ & $<4$ & \\
\hline 7 & $68 / M$ & 50 & $0 \cdot 177$ & 7 & Cholecystectomy \\
\hline 8 & $62 / \mathrm{F}$ & 30 & $0 \cdot 196$ & 9 & \\
\hline 9 & $39 / \mathrm{F}$ & 2 & 0.204 & 11 & Cholecystectomy \\
\hline 10 & $46 / M$ & 10 & 0.223 & 9 & \\
\hline 11 & $53 / \mathrm{F}$ & 5 & 0.273 & 35 & Partial gastrectomy \\
\hline 12 & $30 / \mathrm{F}$ & 10 & 0.287 & 11 & Cholecystectomy \\
\hline 13 & $63 / F$ & 5 & 0.334 & 24 & \\
\hline 14 & $58 / \mathrm{M}$ & 32 & 0.335 & 32 & Partial gastrectomy \\
\hline 15 & $43 / \mathrm{F}$ & 12 & $0 \cdot 337$ & 51 & Lactose intolerance \\
\hline 16 & $57 / \mathrm{F}$ & 3 & 0.345 & 11 & \\
\hline 17 & $53 / \mathrm{F}$ & 10 & 0.374 & 16 & \\
\hline 18 & $24 / F$ & 5 & 0.403 & 11 & \\
\hline 19 & $69 / \mathrm{F}$ & 9 & $0 \cdot 421$ & 63 & Cholecystectomy, radiation therapy \\
\hline 20 & $58 / \mathrm{F}$ & 20 & 0.454 & 101 & Cholecystectomy, radiation therapy \\
\hline 21 & $54 / \mathrm{F}$ & 6 & 0.466 & 97 & \\
\hline 22 & $50 / \mathrm{F}$ & 2 & 0.576 & 40 & Cholecystectomy \\
\hline 23 & $76 / M$ & 30 & 0.654 & 44 & Cholecystectomy \\
\hline 24 & $29 / M$ & 3 & 0.729 & 103 & \\
\hline 25 & $63 / F$ & 6 & 0.746 & 111 & $\begin{array}{l}\text { Cholecystectomy, villous atrophy of } \\
\text { ileum, lymphocytic colitis }\end{array}$ \\
\hline 26 & $35 / M$ & 3 & $0 \cdot 782$ & 45 & Ileocaecal resection \\
\hline 27 & $68 / F$ & 3 & $1 \cdot 140$ & 57 & $\begin{array}{l}\text { Cholecystectomy, villous adenoma of } \\
\text { ileum }\end{array}$ \\
\hline 28 & $38 / \mathrm{M}$ & 6 & $1 \cdot 964$ & 170 & Extensive ileal resection \\
\hline \multicolumn{2}{|c|}{ Normal values } & & $<0.335$ & $<30$ & \\
\hline
\end{tabular}

$\star$ For SeHCAT controls $(n=29)$, fractional catabolic rate $(\mathrm{FCR})=$ mean $(\mathrm{SEM}) 0 \cdot 225(0 \cdot 020)$ pools/ day. and $65 \%$ aqueous methanol, $7 \alpha$-hydroxy-4cholesten-3-one was eluted with hexane: chloroform, 95:5 (v/v). Analysis was performed by high performance liquid chromatography (HPLC) on a column $(250 \times 4.5 \mathrm{~mm})$ of LiChrospher (Hibar, Si 100, $5 \mu \mathrm{m}$, MerckDarmstadt) connected to a UV detector (254 nm) with hexane:isopropanol, $95: 5 \mathrm{vol} / \mathrm{vol}$ as the mobile phase. ${ }^{3} \mathrm{H}$ Labelled 25 -hydroxyvitamin $\mathrm{D}_{3}$ added before extraction and determined in the effluent from the HPLC column, served to correct for losses and differences in injection volumes.

STATISTICAL ANALYSIS

Correlations were evaluated by the calculation of Spearman's correlation coefficient $\left(\mathbf{R}_{\mathrm{s}}\right)$.

\section{Results}

Patients included in the present study had diarrhoea that in some cases was of long duration. They had all been extensively studied to evaluate the cause of their diarrhoea and the possibility of bile acid malabsorption was studied by the SeHCAT test. The result of the SeHCAT test was then compared with the serum concentration of $7 \alpha$-hydroxy-4-cholesten-3-one. The table summarises the clinical data and results.

There was a wide range of SeHCAT fractional catabolic rates, with 15 patients displaying abnormal values (FCR $\geqslant 0.335$ pools/d) indicating bile acid malabsorption. Twelve patients had both a pathological SeHCAT test and an increased serum concentration of $7 \alpha$-hydroxy-4cholesten-3-one. Thirteen patients showed a normal SeHCAT test, and 11 of these had normal concentrations of $7 \alpha$-hydroxy-4cholesten-3-one. Thus three patients $(16,17,18)$ had borderline pathological SeHCAT tests but normal $7 \alpha$-hydroxy-4-cholesten-3-one concentrations, and two patients $(4,11)$ with normal SeHCAT tests displayed a slightly increased concentration of the bile acid intermediate. There was a highly significant positive correlation $\left(R_{s}=0.80, p<0.001\right.$; fig 2$)$ between the two tests.

Ten of the patients were cholecystectomised, four of whom had additional intestinal pathology. For the cholecystectomised patients without intestinal pathology, four $(2,7,9,12)$ had normal SeHCAT elimination and $7 \alpha$-hydroxy-4cholesten-3-one values whereas two $(22,23)$ had bile acid malabsorption and a slight increase in bile acid synthesis as measured by the bile acid intermediate.

Twelve of the patients, including. 10 with pathological SeHCAT results, were treated with cholestyramine. Eight responded well whereas four patients including the two with normal SeHCAT values did not respond. The other two patients who did not show a positive response had lymphocytic colitis (patient 25) and extensive ileal resection (patient 28).

SERUM

$7 \alpha$-Hydroxy-4-cholesten-3-one was analysed as described previously. ${ }^{8}$ Briefly, serum $(1 \mathrm{ml})$ was diluted with two volumes of isotonic saline and extracted on a small column of octadecysilane bonded silica at $64^{\circ} \mathrm{C}$. After a wash with water

\section{Discussion}

Bile acid malabsorption may be involved in the development of chronic diarrhoea in disorders of 


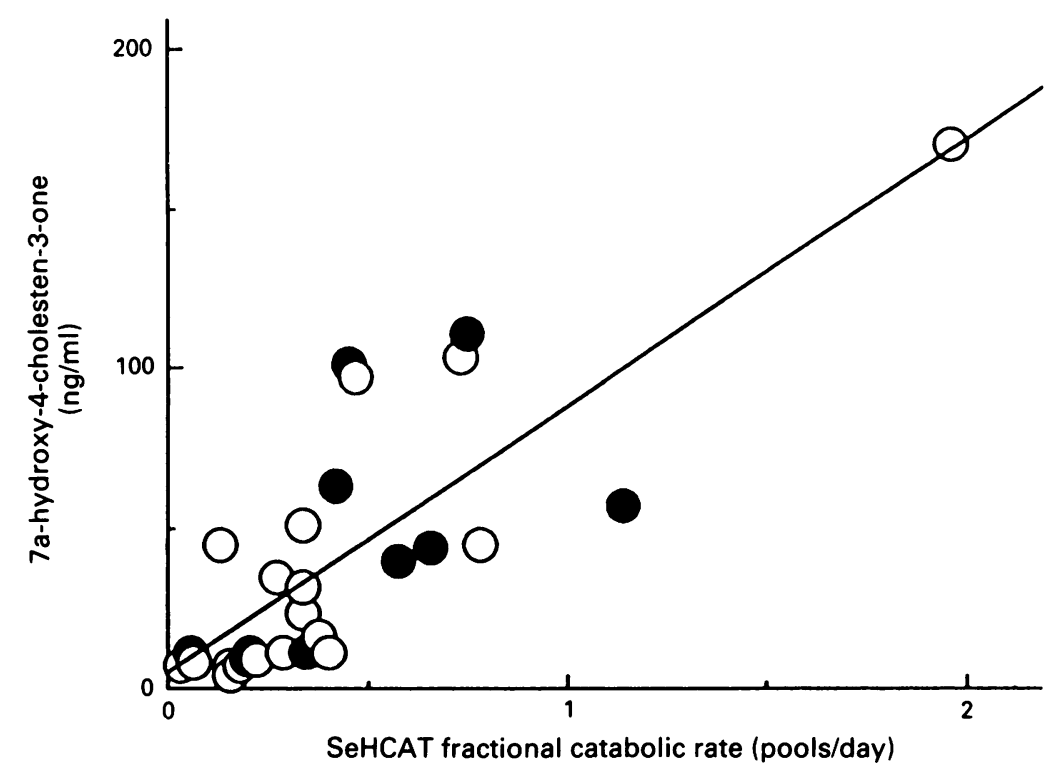

Figure 2: Serum concentration of 7 $\alpha$-hydroxy-4-cholesten-3-one v SeHCAT fractional catabolic rate in the patients $\left(R_{\mathrm{s}}=0 \cdot 80, p<0.001\right)$. Closed circles denote cholecystectomised patients. could so happen that slight bile acid malabsorption may or may not affect bile acid synthesis. It is also possible that small intestinal overgrowth with deconjugating bacteria was present in some cases, which could counteract a concomitant effect of ileal dysfunction on bile acid synthesis. Such phenomena may have been present in three of our patients, in whom a slight bile acid malabsorption was found with the SeHCAT test, but in whom there was no increase in bile acid synthesis by the liver. On the other hand, the SeHCAT value corresponds to a seven day elimination of bile acids, whereas the concentration of $7 \alpha$-hydroxy-4-cholesten-3-one only reflects the synthesis at a certain time. As the concentration of $7 \alpha$-hydroxy-4-cholesten-3-one can vary during the day and acute increases have been found, a better correlation may have been obtained if a serum sample had been taken at more than one occasion from each patient. Such diurnal variations may possibly explain why two of the patients showed increased bile acid synthesis but normal SeHCAT values. As substantial interindividual differences in synthesis of bile acids exist, it may be expected that the SeHCAT test could be more sensitive in evaluation of ileal uptake, especially in the borderline case. At any rate, there were no discrepancies among the patients showing grossly abnormal values for either test.

Both the tests showed similar results in the cholecystectomised patients. Bile acid malabsorption and increased synthesis of bile acids were even detected in cholecystectomised patients without intestinal pathology. Contrary to what had been suggested by a previous report, ${ }^{12}$ the SeHCAT test may thus be of value also in cholecystectomised patients.

In conclusion, there was a strong positive correlation between circulating concentrations of $7 \alpha$-hydroxy-4-cholesten-3-one and values obtained with the SeHCAT test in patients with diarrhoea. This suggests that the determination of the serum concentration of $7 \alpha$-hydroxy-4cholesten-3-one may be useful for the assessment of bile acid malabsorption in such patients. Future prospective studies should now evaluate the clinical relevance of this method, which, through its analytical simplicity and limited patient efforts, should be open to wide use. tions where there is bile acid loss - for example, after ileal resection and cholestyramine treatment, and decreases where there is decreased bile acid synthesis - for example, in cirrhosis of the liver. ${ }^{8}$

In this study we found a good agreement between the two tests, particularly considering the fact that they measure two different variables - namely, bile acid losses and bile acid synthesis. The advantages in determining serum $7 \alpha-$ hydroxy-4-cholesten-3-one concentration are that it is an even simpler method, that only a serum sample is needed, that no radioactivity needs to be used, and that it is less time consuming.

There were a few discrepancies in the results. It has previously been found that an enhanced bile acid loss may also be the consequence of diarrhoea and increased intestinal transit itself, and bile acid malabsorption is not often likely to be the primary cause of the diarrhoea. ${ }^{17-20} \mathrm{It}$
The skilful technical assistance of Ms Pia Gustafsson, Ms Kristina Garmark, and Ms Kerstin Nauclér, and the manuscript preparation of Ms Lena Ericsson are gratefully acknowledged. This study was supported by the Swedish Medical Research Council (03X-
$7890,03 X-7137,03 X-4793)$, the Axel and Margaret Ax:son 7890, 03X-7137, 03X-4793), the Axel and Margaret Ax:son
Johnson Foundation, and the Karolinska Institute. Dr Eusufzai is the recipient of a scholarship from the Swedish Institute.

1 Carey MC, Cahalane MJ. Enterohepatic circulation. In: Arias IM, Jakoby WB, Popper H, Schachter D, Shafritz DA, eds. The liver:biology and pathology. New York: Raven Press, 1988: 573-616.

2 Merrick MV, Eastwood MA, Anderson JR, Ross HM. Enterohepatic circulation in man of a gamma-emitting bile acid conjugate, 23-selena-25 homocholic acid taurine (SeHCAT). F Nucl Med 1982; 23: 126-30.

3 Thaysen EH. Assessment of ileal function by abdominal counting of the retention of a gamma emitting bile acid analogue. Gut 1982; 23: 862-5.

4 Galatola G, Jazrawi RP, Bridges C, Joseph AEA, Northfield TC. Hepatic handling of the synthetic-labelled bile acid SeHCAT. Gastroenterology 1988; 94: 771-8.

5 Delhez $H$, van den Berg JWO, van Blankenstein $M$, acid turnover using ${ }^{75} \mathrm{Se}$-homocholic acid taurine. Eur $\mathcal{F}$ Nucl Med 1982; 7: 269-71. tion of Ms Lena Ericsson are gratefully acknowledged. This study
was supported by the Swedish Medical Research Council (03XMeerwaldt JH. New method for the determination of bile 
6 Jazrawi RP, Ferraris R, Bridges C, Northfield TC. Kinetics for synthetic bile acid ${ }^{75}$ Selenohomocholic acid taurine in humans: comparison with $\left[{ }^{14} \mathrm{C}\right]$ taurocholate. Gastroenterology 1988; 95: 164-9.

7 Björkhem I. Mechanism of bile acid biosynthesis in mammalian liver. In: Danielsson H, Sjövall J, eds. Sterols and bile acids. Comprehensive biochemistry. Amsterdam: Elsevier, 1985: 231-78.

8 Axelson $M$, Aly A, Sjövall J. Levels of $7 \alpha$-hydroxy-4cholesten-3-one in plasma reflect rates of bile acid synthesis cholesten-3-one in plasma reflect rates

9 Axelson M, Björkhem I, Reihnér E, Einarsson K. The plasma level of $7 \alpha$-hydroxy-4-cholesten-3-one reflects the acivity of hepatic cholesterol $7 \alpha$-hydroxylase in man. FEBS Lett 1991; 284: 216-8.

10 Eusufzai S, Ericsson S, Cederlund T, Einarsson K, Angelin B. Effect of ursodeoxycholic on ileal absorption of bile acids in man as determined by the SeHCAT test. Gut 1991; 32: 1044-8.

11 Fromm $\mathrm{H}$, Malavolti $M$. Bile acid-induced diarrhoea. Clinical Gasteroenterology 1986; 15: 567-82.

12 Suhr O, Danielsson $\AA$, Nyhlin H, Truedsson H. Bile acid malabsorption demonstrated by SeHCAT in chronic diarrhoea, with special reference to the impact of diarrhoea, with special reference to the impact
cholecystectomy. Scand $\mathcal{G}$ Gastroenterol 1988; 23: 1187-94.

13 Condon JR, Robinson V, Suleman MI, Fan VS, Mckeown MD. The cause and treatment of post vagotomy diarrhoea. BMF 1985; 290: 1298-9.
14 Williams AJK, Merrick MV, Eastwood MA. Idiopathic bile acid malabsorption - a review of clinical presentation, diagnosis and response to treatment. Gut 1991; 32: 1004-6.

15 Hofmann AF, Poley JR Role of bile acid malabsorption in the pathogenesis of diarrhoea and steatorrhea in patients with ileal resection. Response to cholestyramine or replacement of dietary long chain triglyceride by medium chain triglyceride. Gastroenterology 1972; 62: 918-34.

triglyceride. Gastroenterology 1972; 62: 918-34.
16 Mekhjian MS, Phillips SF, Hofmann AF. Colonic secretion of water and electrolytes induced by bile acid perfusion studies water and electrolytes induced by bile acid

17 Fromm H, Thomas PJ, Hofmann AF. Sensitivity and specificity in tests of distal ileal function: prospective comparison of bile acid and vitamin $\mathbf{B}_{12}$ absorption in ileal resection patients. Gastroenterology 1973; 64: 1077-90.

18 McJunkin B, Fromm H, Sarva RP, Amin P. Factors in the mechanism of diarrhoea in bile acid malabsorption: fecal pH - a key determinant. Gastroenterology 1981; 80: 1454-64.

19 Schiller LR, Hogan RB, Morawski SG, Santa Ana CA, Bern MJ, Norgaard RP, et al. Studies of the prevalence and significance of radiolabeled bile acid malabsorption in a group of patients with idiopathic chronic diarrhoea. Gastroenterology 1987; 92: 151-60.

20 Schiller LR, Bilhartz LE, Santa Ana CA, Fordtran IS. Comparison of endogenous and radiolabeled bile acid excretion in patients with idiopathic chronic diarrhoea. Gastroenterology 1990; 98: 1036-43. 